\title{
Finding the Evidence: Localization-aware Answer Prediction for Text Visual Question Answering
}

\author{
Wei Han* \\ MediaTek, Singapore \\ Wei.Han@mediatek. com
}

\author{
Hantao Huang* \\ MediaTek, Singapore \\ Hantao. Huang@mediatek.com
}

\author{
Tao Han \\ MediaTek, Singapore \\ TaoH.Han@mediatek.com
}

\begin{abstract}
Image text carries essential information to understand the scene and perform reasoning. Textbased visual question answering (text VQA) task focuses on visual questions that require reading text in images. Existing text VQA systems generate an answer by selecting from optical character recognition (OCR) texts or a fixed vocabulary. Positional information of text is underused and there is a lack of evidence for the generated answer. As such, this paper proposes a localizationaware answer prediction network (LaAP-Net) to address this challenge. Our LaAP-Net not only generates the answer to the question but also predicts a bounding box as evidence of the generated answer. Moreover, a context-enriched OCR representation (COR) for multimodal fusion is proposed to facilitate the localization task. Our proposed LaAP-Net outperforms existing approaches on three benchmark datasets for the text VQA task by a noticeable margin.
\end{abstract}

\section{Introduction}

Visual Question Answering (VQA) has attracted much interest from the communities and witnessed tremendous progress. However, lacking the ability to generate answers based on texts in the image limits its applications. Recently, many new datasets (Biten et al., 2019a; Singh et al., 2019) and new methods (Singh et al., 2019; Hu et al., 2020) are proposed to tackle this challenge and refer it as text VQA.

The earliest method for text VQA is LoRRA(Singh et al., 2019), which provides an optical character recognition (OCR) module for the VQA input and proposes a dynamic copy mechanism to select the answer from both fixed vocabulary and OCR words. The following work M4C(Hu et al., 2020) inspired by LoRRA, uses rich representations of OCR as input and utilizes dynamic pointer network to deal with out-of-vocabulary answers, leading to state-of-the-art performance. However, M4C simply concatenates all modalities as transformer input and does not consider the high-level interaction among modalities of text VQA. Moreover, it is unable to provide evidence for the answer since the text is not localized in the image. Another recent work (Wang et al., 2020) proposes a new dataset for evidence-based text VQA, which suggests Intersection over Union (IoU) based evaluation metric to measure the evidence. Our work follows the spirit of evidence-based text VQA. More specifically, we generate the answer text bounding box during the answer prediction process as supplementary evidence for our answer. We propose a localization-aware answer prediction module (LaAP) that integrates the predicted bounding box with our semantic representation for the final answer. Besides, we propose a multimodal fusion module with context-enriched OCR representation, which uses a novel position-guided attention to integrate context object features into OCR representation.

The contributions of this paper are summarized as follows: 1) We propose a LaAP module, which predicts the OCR position and integrates it with the generated answer embedding for final answer prediction. 2) We propose a context-enriched OCR representation (COR), which enhances the OCR modality and simplifies the multimodal input. 3) We show that the predicted bounding box can provide evidence for analyzing network behavior in addition to improving the performance. 4) Our proposed LaAP-Net

* These authors contributed equally to this work

This work is licensed under a Creative Commons Attribution 4.0 International Licence. Licence details: http:// creativecommons.org/licenses/by/4.0/. 
outperforms state-of-the-art approaches on three benchmark text VQA datasets, TextVQA(Singh et al., 2019), ST-VQA(Biten et al., 2019b) and OCR-VQA(Mishra et al., 2019), by a noticeable margin.

\section{Related Works}

\subsection{Text Visual Question Answering}

Text VQA has attracted much attention from the communities. The predominate method is LoRRA (Singh et al., 2019), which takes image features, OCR features and questions to generate the answer. LoRRA mimics the human answering process by providing the image-looking module, text-reading module and answer-reasoning module. The generated answer could be selected from a fixed answer vocabulary or one of the OCR tokens by the copy module. The copy module is further improved by M4C (Hu et al., 2020) using dynamic pointer network. The M4C also proposes a transformer based network with 3 multi-modal input (question, image object features and OCR features). We share the same spirit as M4C but split the network into a clear encoder-decoder structure. We further propose a context-enriched OCR representation to extract OCR related image features.

\subsection{Evidence-based VQA and Multitask Learning}

Evidence-based VQA has been proposed in the recent work (Wang et al., 2020), which suggests to use intersection over union (IoU) to indicate the evidence. Many existing works (Selvaraju et al., 2017; Goyal et al., 2016; Yu et al., 2019; Gao et al., 2019) compute the attention scores and build spatial maps on image to highlight regions, which the model focuses on. The spatial maps serve as an evidence and visual explanations of a VQA architecture. Our method further extends this by designing a location predictor to generate a bounding box on the image to explain the answer generated. The bounding box explains that the correct answer generated is based on the analysis of underlying reasoning instead of exploiting the statistics of the dataset. As such, the bounding box becomes evidence of the VQA answer. To achieve the aforementioned target, we design a multitask learning process, which not only generates the answer based on the image and question but also provides the bounding box for the answer. The proposed method improves the interpretation of VQA results and leads to better performance.

\section{Localization-aware Answer Prediction Network}

\subsection{LaAP Network Architecture}

To better utilize the position information of image texts and enforce the network to better exploit visual features, we propose a localization-aware answer prediction network (LaAP-Net). Our LaAP-Net is built based on the multimodal transformer encoder, transformer decoder and localization-aware prediction network as shown in Figure 1. The transformer encoder takes the question embedding and OCR embedding as input. Question embedding is generated by putting the question through a pretrained BERT-based model, whereas the OCR embedding is generated by our proposed context-enriched OCR representation module. As highlighted in dark yellow in Figure 1, the decoding process starts with the <begin > signal. For each decoded output, we first generate a bounding box. This bounding box will then be embedded and added to the current answer decoder output, which is referred as localization-aware answer representation. Finally, it is fed to the vocabulary score module and OCR score module. The scores are concatenated and the element with the maximum score is selected as the final answer. In the following section, we will present the three components of LaAP-Net: the context-enriched OCR representation, the localization-aware predictor and the transformer with simplified decoder.

\subsection{Context-enriched OCR Representation}

Existing work (Hu et al., 2020) builds a common embedding space for all modalities. However, this common embedding space has difficulty utilizing the image object features. We observe this by training the M4C (Hu et al., 2020) network without the image object modality. The accuracy is almost unaffected. To better exploit the image object modality, we propose the context-enriched OCR representation (COR) module (Shown in Figure 2). Ideally, the answer for text VQA should be found from OCR tokens, thus we 


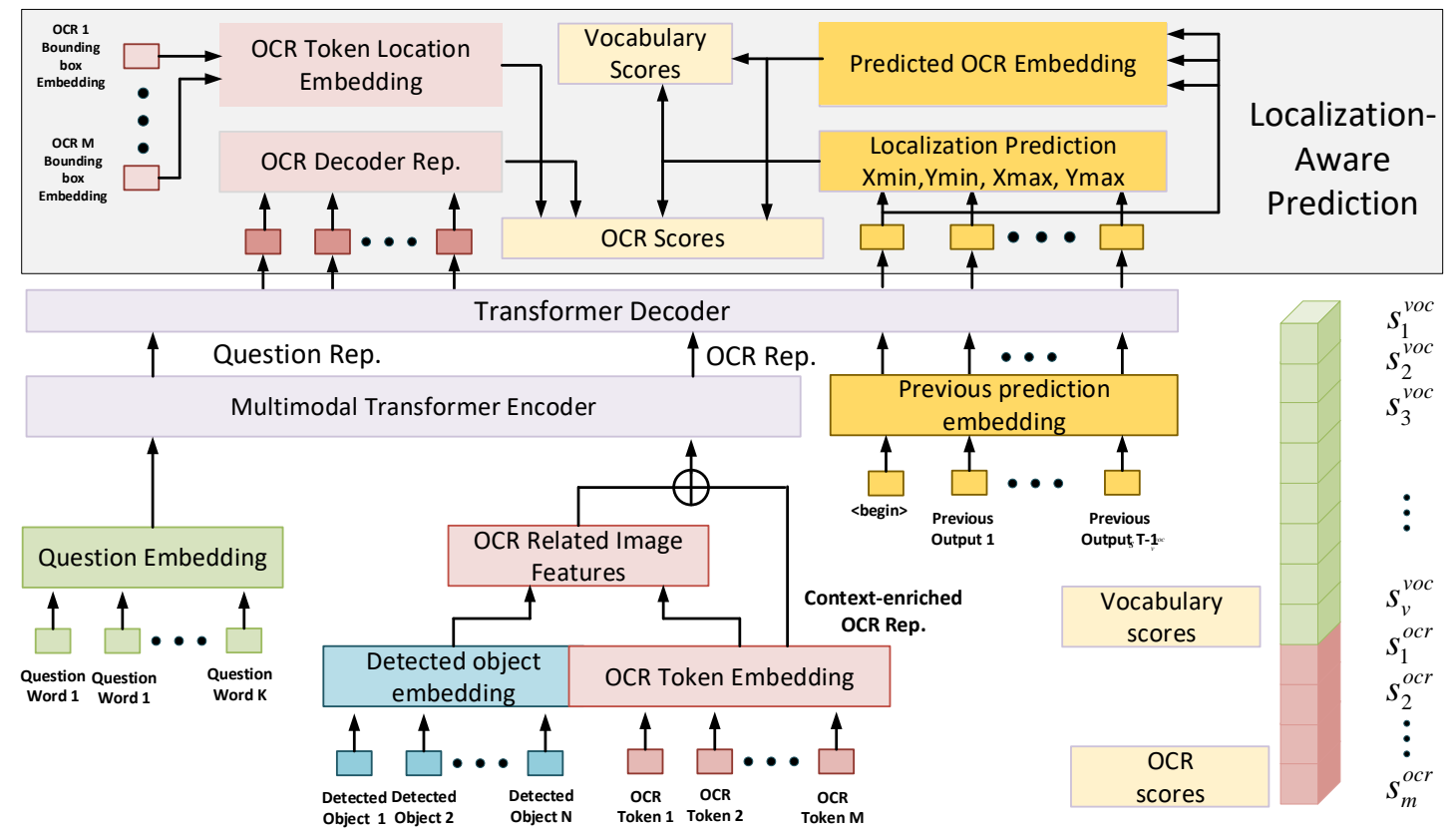

Figure 1: An overview of LaAP model. We perform context-enriched OCR representation to extrat object features. Then question words and enriched OCR tokens are input to the transformer encoder and the transformer decoder. Based on the transformer decoder outputs, we first predict the answer localization, and then integrate this localization to the OCR embedding. Decoder output is also equipped with OCR position embedding. The OCR scores and vocabular scores are calculated accordingly to find the answer from an OCR token or a word from the fixed answer vocabulary.

integrate geometric context objects of an OCR token into its representation to improve the discriminative power. Take Figure 5(b) for example, the OCR representation for sixers enriched with the features of the object red jersey can be better attended by the question. The context objects are attended according to the proposed position-guided attention, where only spatial relationship between objects and OCR tokens are considered.

Following M4C (Hu et al., 2020), we use features extracted from $N$ object detected by the Faster R-CNN (Ren et al., 2015), denoted as $x_{n}^{o b j}$ (where $\left.n=1, \ldots, N\right)$. The corresponding bounding box coordinates are represented as $b_{n}^{o b j}$ (where $\left.n=1, \ldots, N\right)$. A combination of Faster R-CNN, Pyramidal Histogram of Characters (PHOC) (Almazán et al., 2014) and FastText (Bojanowski et al., 2017) embedding is adopted for $M$ OCR tokens in an image, denoted as $x_{m}^{o c r}$ (where $m=1, \ldots, M$ ) with the bounding box denoted as $b_{m}^{o c r}$ (where $\left.m=1, \ldots, M\right)$. We embed the given question into a set of word embedding $x_{k}^{\text {ques }}$ (where $k=1, \ldots, K$ and $K$ is the number of words) through a pretrained BERT language model (Devlin et al., 2019). All embeddings are then linearly projected to a $d$-dimensional space.

The detailed computation process for COR is described as follows. Firstly, the position-guided attention score vector $a t t_{m}$ between the $m$-th OCR token and the image objects is calculated as

$$
a t t_{m}=\operatorname{softmax}\left(\left(W^{Q} b_{m}^{o c r}\right)^{T} *\left[W^{K} b_{1}^{o b j}, \ldots, W^{K} b_{N}^{o b j}\right]\right), m=1, \ldots, M
$$

where $W^{Q}$ and $W^{K}$ are query projection matrix and key projection matrix respectively. Then the $m$-th image attended OCR representation is calculated as weighted sum of the $N$ object feature vectors as

$$
x_{m}^{o c r \mid o b j}=\left[x_{1}^{o b j}, \ldots, x_{N}^{o b j}\right] * a t t_{m}^{T}, m=1, \ldots, M
$$

Note that we omit the multi-head attention mechanism (Vaswani et al., 2017) for simplicity. Finally, each OCR token is represented by aggregating OCR feature embedding, image attended OCR representation and position embedding as

$$
\hat{x}_{m}^{o c r}=x_{m}^{o c r}+x_{m}^{o c r \mid o b j}+W^{o c r} b_{m}^{o c r}, m=1, \ldots, M
$$




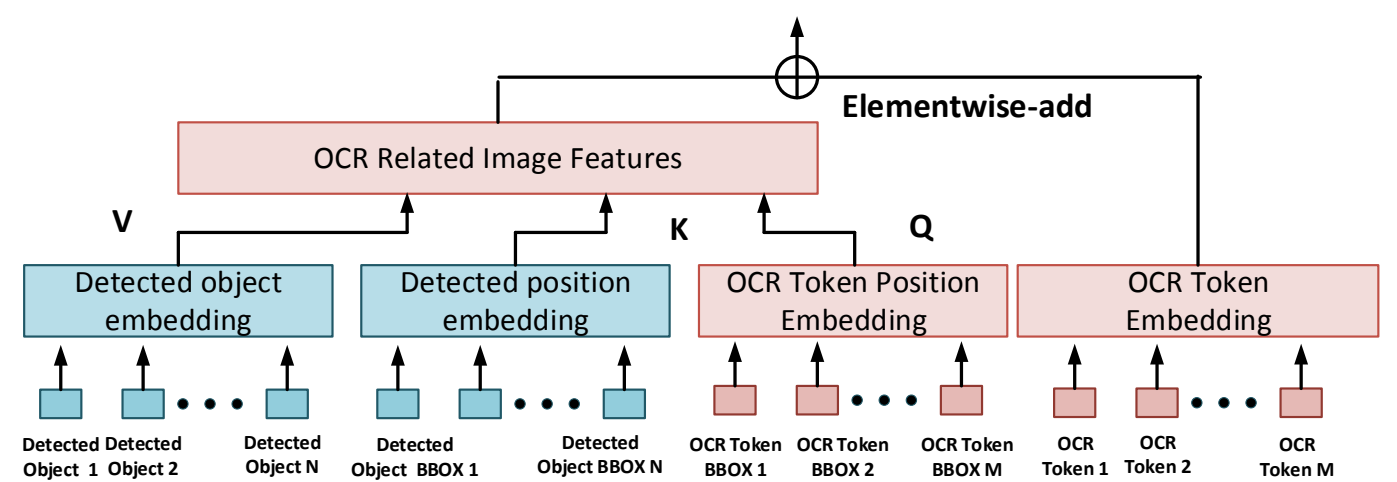

Figure 2: Context-enriched OCR representation to integrate the object features based on the spatial information (bounding box)

where $W^{o c r}$ is a matrix that linearly project the bounding box coordinate vector to $d$ dimension. With the proposed attention, the image object modality is merged into OCR. We then feed $\hat{x}_{1}^{\text {ocr }}, \ldots, \hat{x}_{M}^{\text {ocr }}$ and $x_{1}^{q u e s}, \ldots, x_{K}^{q u e s}$ into the transformer encoder as input. The strengthened OCR representation $\hat{x}_{m}^{o c r}$ empowers the network to better learn the semantic correlation between OCR tokens and question. Meanwhile, it simplifies the multimodal feature input to improve the localization-aware answer prediction.

\subsection{Localization-aware Answer Prediction}

\subsubsection{Localization-aware Predictor}

To exploit the positional information of image features and texts, we design a localization-aware predictor to perform the bounding box prediction. The bounding box is embedded and added to the decoder output to generate the localization-aware answer representation. More specifically, given the answer embedding $y^{d e c}$ output from the decoder, we calculate the localization-aware answer representation $z^{\text {ans }}$ by fusing $y^{d e c}$ with the gated bounding box projection as

$$
z^{a n s}=y^{d e c}+g^{l o c} \circ\left(W^{l o c} * b^{p r e d}+b i a s^{l o c}\right)
$$

where $W^{l o c}$ and bias ${ }^{l o c}$ are weights of a linear layer to project the location bounding box to the same dimension as $y^{d e c}$ and $\circ$ represents element-wise multiplication. $g^{l o c}$ is the localization gate. Note that our network update the gate weight automatically through training, so that it implicitly reveals the statistical importance of the localization information. Similarly, we calculate the high-level localizationaware representation $z_{m}^{o c r}$ (where $m=1, \ldots M$ ) of each OCR token as

$$
z_{m}^{o c r}=y_{m}^{o c r}+g^{l o c} \circ\left(W^{l o c} * b_{m}^{o c r}+b i a s^{l o c}\right), m=1, \ldots, M
$$

where $y_{m}^{o c r}$, denotes the m-th OCR encoding from the last encoder layer and $b_{m}^{o c r}$ is the corresponding bounding box coordinates. $b_{m}^{o c r}$ goes through the same linear projection layer and localization gate as $b^{\text {pred }}$ so that they are projected to the same high-dimensional space.

Then similar to (Hu et al., 2020), we obtain the similarity score $s_{m}^{o c r}$ between each OCR representation and the answer representation as

$$
s_{m}^{o c r}=\left(W^{a n s} * z^{a n s}+\text { bias }^{a n s}\right)\left(W^{o c r} * z_{m}^{o c r}+\text { bias }^{o c r}\right), m=1, \ldots, M
$$

where $W^{a n s}$, bias ${ }^{a n s}, W^{o c r}$ and bias ${ }^{o c r}$ are parameters of linear projection layers. The localizationaware answer representation $z^{\text {ans }}$ is also fed into a classifier to output $V$ scores $s_{v}^{v o c}(v=1, \ldots, V)$, where $V$ is the vocabulary size. The final prediction is selected as the element with the maximum score as

$$
\max \left[s_{m}^{o c r} ; s_{v}^{v o c}\right]
$$




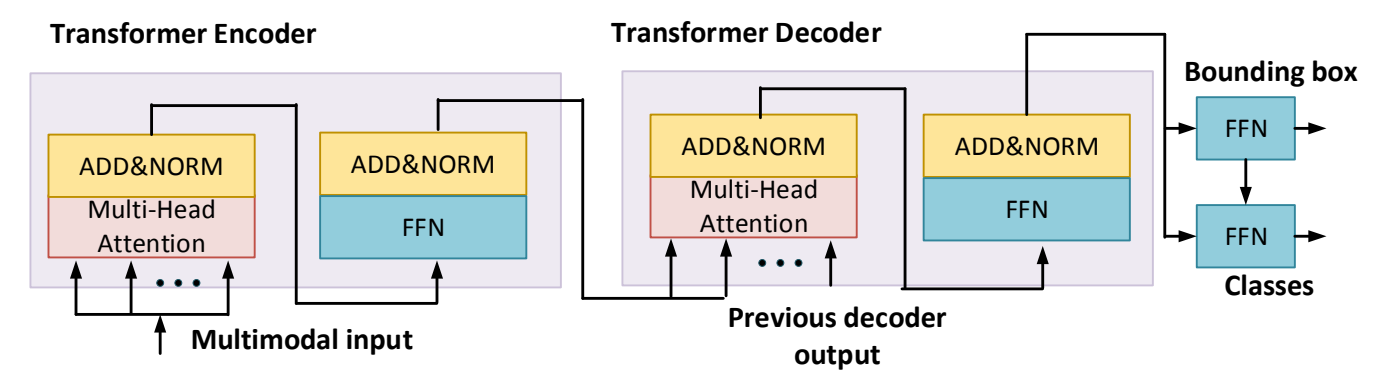

Figure 3: An overview of the transformer with simplified decoder (TSD). TSD output is used to generate the bounding box, which is then used for answer prediction

Note that the predicted bounding box is not explicitly used in generating the answer. However, localization prediction is a vision task so it can enforce the network to exploit visual features. As a result, it serves as a good complement to the classical vocabulary classification task, which mainly focuses on linguistic semantics. The localization-aware predictor strengthens the learned answer embedding to attend to the correct OCR token, which in turn facilitates the classifier to correctly find the word. Moreover, this localization information improves the performance of position-related questions as shown in Figure 4(a) and 4(c), which will be further discussed in Section 4.2

\subsubsection{Loss Design to Incorporate the Evidence Scores}

We use the IoU scores as the evidence for the answer generated. Therefore, we propose a multitask loss, which facilitates the answer embedding to learn both the semantics and localization information provided by the OCR tokens. The proposed multi-task loss consists of three individual loss functions: localization loss $L_{l}$, semantic loss $L_{s}$ and the fusion loss $L_{f}$.

The answer embedding output from the decoder is fed into a multilayer perceptron (MLP) to directly predict the bounding box location $b^{\text {pred }}$ of the answer OCR token. Inspired by (Carion et al., 2020), the localization loss $L_{l}$ is defined as:

$$
L_{l}=\left(1-I o U\left(b^{\text {pred }}, b^{g t}\right)+\mathbb{L}_{1}\left(b^{\text {pred }}, b^{g t}\right)\right) * \mathbb{I}
$$

where $b^{g t}$ denotes the ground truth bounding box, which is obtained by matching the OCR token text to the ground truth answer text. IoU and $\mathbb{L}_{1}$ calculate the intersection over union and $\mathrm{L} 1$ norm respectively between the prediction and ground-truth bounding box. $\mathbb{I}=1$ if the answer word matches one of the recognized OCR text and 0 otherwise.

To accurately answer a question, OCR localization and semantic information are both critical. Thus, we propose a fusion loss $L_{f}$ to couple the localization prediction and semantic representation of the answer. The two aspects of information complement each other in the process of decision making. Formally, given the target scores $t_{m}^{\text {ocr }} \in\{0,1\}(m=1, \ldots M)$, we formulate our fusion loss $L_{f}$ using cross entropy as

$$
L_{f}=\sum_{i=1}^{M} l o s s_{\text {cross_entropy }}\left(s_{m}^{o c r}, t_{m}^{o c r}\right)
$$

In order to exploit the linguistic meaning of the answer embedding, we collect a fixed vocabulary of frequently used words. We feed the localization-aware answer representation $z^{\text {ans }}$ into a linear classifier to classify answer embedding of each decoding step to one of the vocabulary. Our semantic loss $L_{s}$ is computed as the cross entropy between the classification score vector and the one hot encoding from the ground truth word. The overall multi-task loss of the network is calculated as $L=L_{f}+\lambda_{l} L_{l}+$ $\lambda_{s} L_{s}$, where $\lambda_{l}$ and $\lambda_{s}$ are regulation coefficients that determine the importance of localization loss and semantic loss. The value of $\lambda_{l}$ and $\lambda_{s}$ are experimentally selected. 


\subsection{Transformer with Simplified Decoder}

Existing works (Hu et al., 2020; Gao et al., 2020) use BERT alike transformer architecture, which allows each decoder layer to attend to the same depth encoder layer. However, a deeper encoder layer extracts a more broad view of the input than a shallow layer (Clark et al., 2019). As such, we adopt the standard transformer encoder-decoder structure as shown in Figure 3. Here, we use the transformer with simplified decoder (TSD) by removing the decoder self-attention to save the computational cost. We experimentally find that only using the encoder-decoder attention can maintain the same performance. The multimodal inputs are encoded by $L$ stacked standard transformer encoder layers. The embedding of the last encoder layer is fed into each of the $L$ decoder layers. The answer word is generated in an auto-regressive manner, i.e. for each decoding step, we take the predicted answer embedding from the previous step as the decoder input and obtain the answer embedding as the decoder output. The decoding process is performed by the proposed localization-aware prediction module as shown in Figure 1 and discussed in Section 3.3.

\section{Experiments}

We evaluate our LaAP-Net on the three challenging benchmark datasets: TextVQA (Singh et al., 2019), ST-VQA (Biten et al., 2019a) and OCR-VQA (Mishra et al., 2019). We show that the proposed LaAPVQA network outperforms state-of-the-art works on these datasets. We further perform the ablation study to investigate the proposed context-enriched OCR representation (COR) and the localization-aware answer prediction (LaAP) on TextVQA dataset.

\subsection{Implementation Details}

For a fair comparison with the state-of-the-art methods, we follow the same multimodal input as M4C (Hu et al., 2020). More specifically, we use a pretrained BERT (Devlin et al., 2019) model for question encoding, the Rosetta-en OCR system (Borisyuk et al., 2018) for OCR representation and a Faster-RCNN (Ren et al., 2015) based image feature extraction. The OCR tokens are represented by a concatenation of the appearance features from Faster R-CNN , FastText embeddings (Bojanowski et al., 2017), PHOC feature (Almazán et al., 2014) and bounding box (bbox) embedding. We set the common dimensionality $d=768$ and the number of transformer layers $L=4$. More details of training configuration are summarized in the supplementary material.

\subsection{Evaluation on TextVQA Dataset}

The TextVQA (Singh et al., 2019) dataset contains 28408 images with 34602 training, 5000 validation and 5734 testing question-answer pairs. We compare our result on TextVQA to the newest SOTA method SMA (Gao et al., 2020) and other existing works like LoRRA (Singh et al., 2019), MSFT VTI (MSFTVTI, 2019), and M4C (Hu et al., 2020). The proposed LaAP-Net achieves a $40.68 \%$ validation accuracy and a $40.54 \%$ testing accuracy, which improves the SOTA by $1.10 \%$ (absolute) and $0.25 \%$ (absolute).

\begin{tabular}{|c|c|c|}
\hline Methods & Val Acc. & Test Acc. \\
\hline LoRRA & 26.56 & 27.63 \\
\hline M4c & 39.40 & 39.01 \\
\hline M4C+COR & 39.78 & -- \\
\hline M4C+COR+LaP & 40.73 & 40.41 \\
\hline \hline TSD & 39.86 & - \\
\hline TSD+LaP & 39.37 & - \\
\hline TSD+COR & 40.49 & - \\
\hline TSD+COR+LaP(ours) & 40.68 & 40.54 \\
\hline
\end{tabular}

Table 1: Ablation study on context-enriched OCR representation and localization-aware answer prediction for M4C model and our proposed model

\begin{tabular}{|c|c|}
\hline Methods & Val Acc \\
\hline M4C & 39.40 \\
\hline w/o Vocab & 31.76 \\
\hline w/o OCR copy & 14.94 \\
\hline \hline LaAP-Net (ours) & 40.68 \\
\hline w/o Vocab. & 31.37 \\
\hline w/o OCR Copy & 24.71 \\
\hline w/o OCR embedding Copy & 34.51 \\
\hline w/o OCR bbox Copy & 40.49 \\
\hline
\end{tabular}

Table 2: Ablation study by removing its fixed answer vocabulary (w/o Vocab.) or OCR copying (w/o OCR Copy) on the TextVQA dataset. 


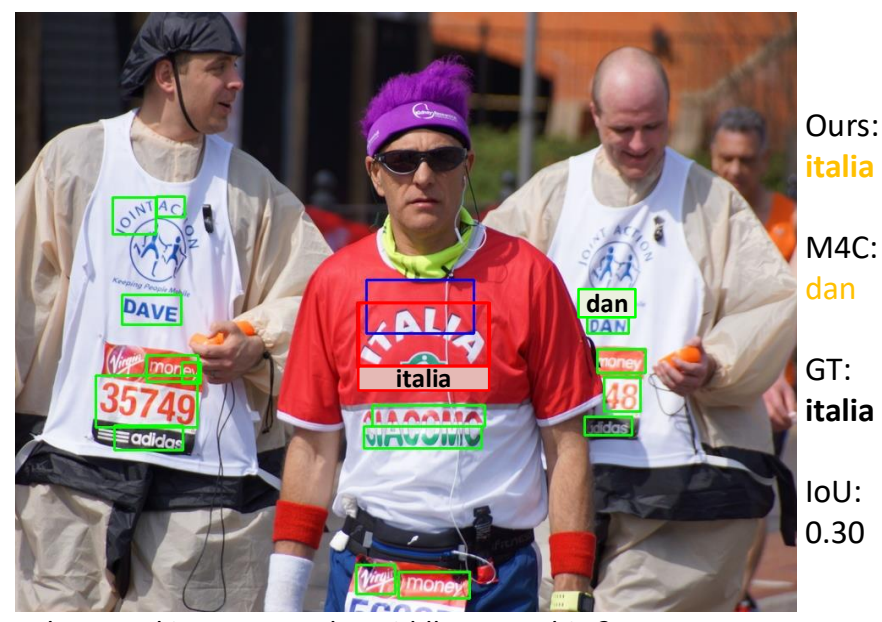

What word is wrote on the middle mans shirt?

(a)

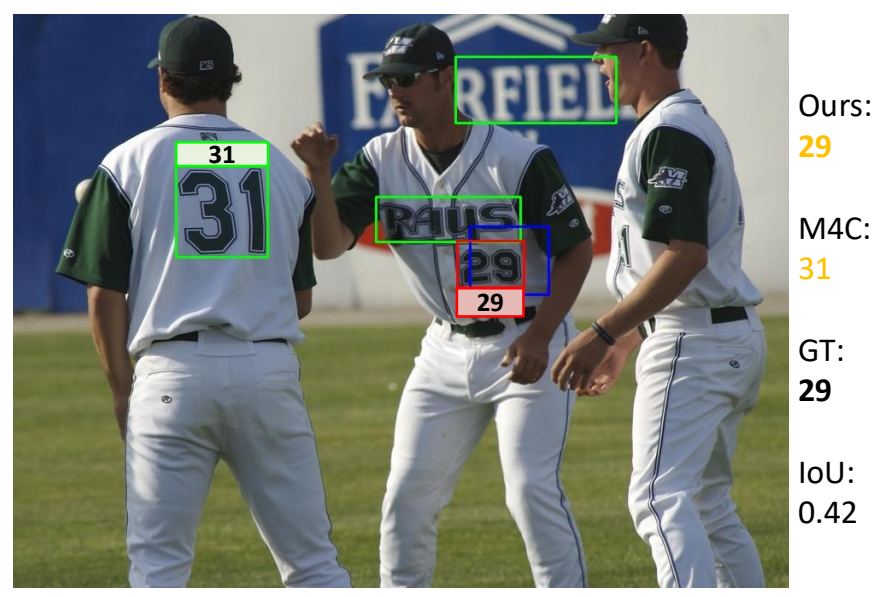

What number of guy in middle?

(c)

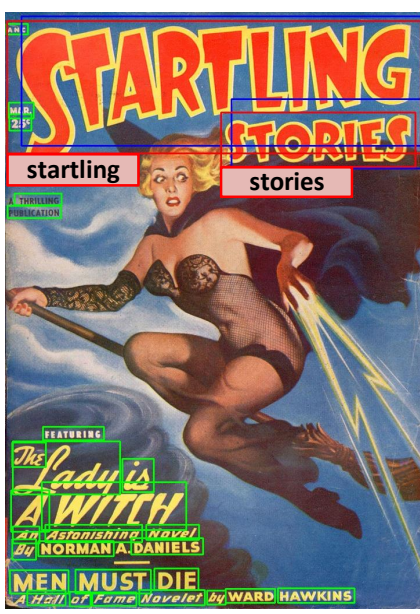

Ours:

startling

stories

M4C:

startling

GT:

startling

stories

IoU:

0.84

0.68

(b)

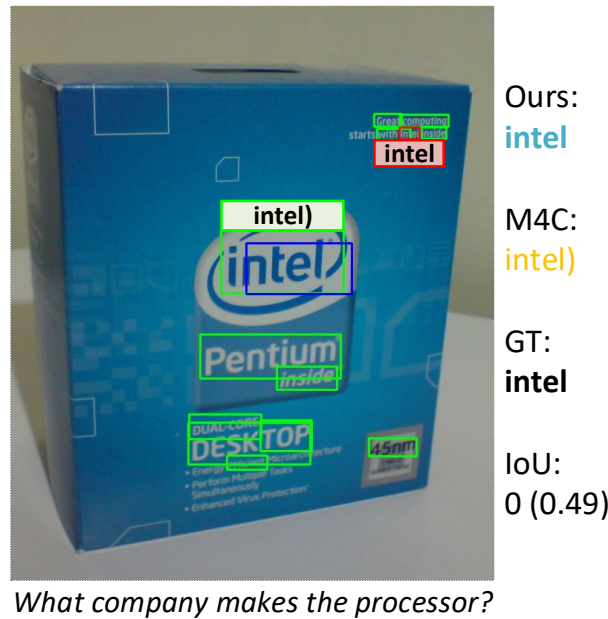

(d)

Figure 4: Qualitative examples from TextVQA dataset. We display predicted answers (Yellow for word generated from OCR and blue for vocabulary) of LaAP-Net and M4C with ground-truth (GT). Our predicted bounding box (blue box) is also depicted in the images to compare to the GT box (red box). The IoU as an evidence score is also shown in each image. For Figure (d), IoU 0 ( 0.49 ) indicates OCR recognition error, 0.49 is the IoU with the GT bounding box.

Note that we only compare with SMA results using the same set of features to show the advantage of the network structure itself. We also train our network with additional data from ST-VQA dataset following M4C and boost the test accuracy by $0.95 \%$ (absolute).

Ablation Study on Network Components. Context-enriched OCR representation (COR) and Localization-aware predictor ( $\mathrm{LaP})$ are the two key features of our network. We investigate the importance of both components by progressively adding them on our transformer with simplified decoder (TSD) backbone. First, we remove COR and LaP from our network and feed image object feature directly into the encoder as in M4C. The answer prediction part is also strictly following M4C. This configuration is denoted as TSD in Table.1. Then we add COR on TSD, which is denoted as TSD+COR. The third ablation is adding only LaP to TSD (TSD+LaP). Each component demonstrates a contribution to performance improvement as shown in Table 1. To further prove the effectiveness of COR and LaP, we add them on our baseline network M4C. COR and LaP individually lead to an accuracy improvement of 0.38 and 0.95 respectively. COR and LaP together boost the accuracy by 1.33 . Note that our network without COR, i.e. TSD+LaP surfers from performance retrogress. The rationale behind is that flat multimodal feature used in place of COR contains both objects and OCR tokens. Object's position embedding introduces much noise for the localization task. COR absorbs context object features in OCR representation and improves its discriminating power. Meanwhile, the encoder multimodal input is sim- 


\begin{tabular}{|c|c|c|}
\hline Method & $\begin{array}{c}\text { Val. } \\
\text { Acc. }\end{array}$ & $\begin{array}{c}\text { Test } \\
\text { Acc. }\end{array}$ \\
\hline LoRRA (Singh et al., 2019) & 26.56 & 27.63 \\
\hline MSFT VTI (MSFT-VTI, 2019) & 32,92 & 32.46 \\
\hline M4C (Hu et al., 2020) & 39.4 & 39.1 \\
\hline SMA (Gao et al., 2020) & 39.58 & 40.29 \\
\hline LaAP-Net & $\mathbf{4 0 . 6 8}$ & $\mathbf{4 0 . 5 4}$ \\
\hline \hline M4C + STVQA (Hu et al., 2020) & 40.55 & 40.46 \\
\hline LaAP-Net + STVQA & $\mathbf{4 1 . 0 2}$ & $\mathbf{4 1 . 4 1}$ \\
\hline
\end{tabular}

Table 3: On the TextVQA dataset, our model outperforms LoRRA and M4C by $13.11 \%$ and $1.44 \%$ (absolute) respectively. Our final model trained on TextVQA and STVQA dataset advances the state-of-the-art performance to $41.41 \%$ on TextVQA test split dataset.

\begin{tabular}{|c|c|c|c|}
\hline Method & $\begin{array}{c}\text { Acc. } \\
\text { Val }\end{array}$ & $\begin{array}{c}\text { ANLS } \\
\text { Val. }\end{array}$ & $\begin{array}{c}\text { ANLS } \\
\text { Test }\end{array}$ \\
\hline $\begin{array}{c}\text { VTA } \\
\text { (Biten et al., 2019a) }\end{array}$ & - & - & 0.282 \\
\hline $\begin{array}{c}\text { M4C } \\
\text { (Hu et al., 2020) }\end{array}$ & 38.05 & 0.472 & 0.462 \\
\hline $\begin{array}{c}\text { SMA } \\
\text { (Gao et al., 2020) }\end{array}$ & - & - & 0.466 \\
\hline LaAP-Net & 39.74 & 0.4974 & $\mathbf{0 . 4 8 5}$ \\
\hline
\end{tabular}

Table 4: On the STVQA datset, our LaAP-Net model achieves +0.02 (absolute) ANLS over the most recent work SMA and approximately +0.2 (absolute) boost over the challenge winner, VTA (Biten et al., 2019a).

plified, which leads to noise reduction. In summary, LaP and COR are not two independent modules simply added together. They enhance each other and improve our network as a system.

Ablation Study on Source of Answer. We restrict the answer generation source to study the effect of our method on word semantic learning and OCR selection. As shown in Table.2, our model significantly improves the accuracy when we only predict the answer from vocabulary. It implies that our localization prediction module enhances the network's capacity for learning the semantics of OCR tokens, which coincides with our qualitative analysis.

Evidence-based Qualitative Analysis on TextVQA Dataset. One challenge for the existing VQA system is that the correct answer generated is hard to tell whether the answer is based on the analysis of underlying reasoning or through exploiting the statistics of the dataset. As such, Intersection-over-Union (IoU) (Wang et al., 2020) is recommended to measure the evidence for the answer generated. The IoU result of our bounding box is shown in Figure 4. For example, in Figure 4(b), two IoU results $(0.84,0.68)$ explain the reason for the answer "startling stories". Higher IoU indicates better evidence. Furthermore, these IoU scores show the answer is generated by exploiting the image features instead of exploiting the statistics of the data set, i.e. a coincidental correlation in the data.

Furthermore, we observe that most of the text VQA errors come from inaccurate OCR result. e.g. in Figure 4(d), the OCR token "intel)" is recognized wrongly, which results in the false answer of M4C. Due to the localization prediction, our method generates the correct answer even in such case (4(d)). Since localization tends to use visual features of OCR tokens rather than their text embedding, it can better determine the attended OCR token in spite of the text recognition result. With the predicted OCR bounding box, the answer generation problem is converted to a conditioned classification process $P$ (text|predicted box) to recognize the text from the vocabulary. More examples supporting our analysis can be found in Figure 4.

Our localization predictor also shows the capability of understanding position and direction as shown in Figure 4(a, c). Our network learns to understand position in training because the ground-truth position is provided straight to guide the localization prediction, while in previous works, positional information is put through several layers of encoder and decoder without explicit guidance.

\subsection{Evaluation on ST-VQA Dataset.}

We evaluate the proposed model on the open vocabulary task of ST-VQA (Biten et al., 2019b), which contains 18921 training-validation images and 2971 test images. Following previous works (Hu et al., 2020; Gao et al., 2020), we split the images into training and validation set with size of 17028 and 1893 respectively.

We report both accuracy and ANLS score (default metric of ST-VQA) in Table 4. Our LaAP-Net surpasses the SOTA method by a large margin on both metrics. Note that SMA improves its baseline method M4C by only 0.004 in testing ANLS score while we boost the result by 0.019 .

Evidence-based Qualitative Analysis on ST-VQA Figure 5 shows IoU scores, our predicted bounding 


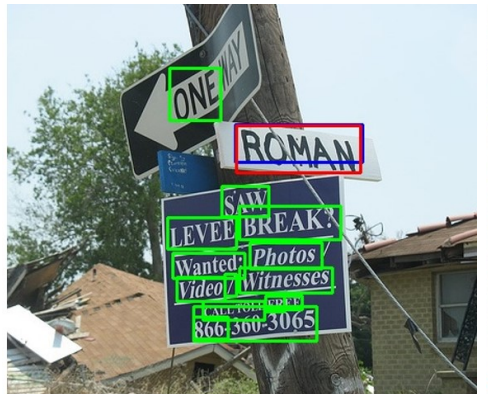

What does the middle sign say?

Ours: roman

M4C: levee

GT: roman

IoU: 0.75

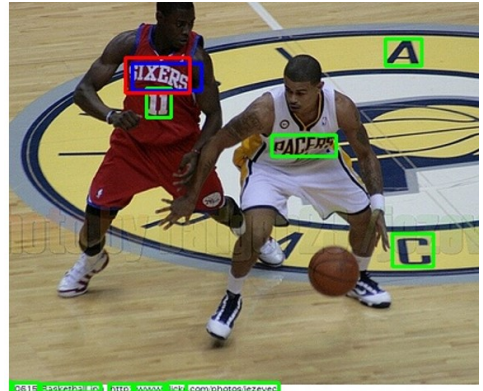

What team is playing in a red jersey?

Ours: sixers

M4C: indiana

GT: sixers

IoU: 0.61

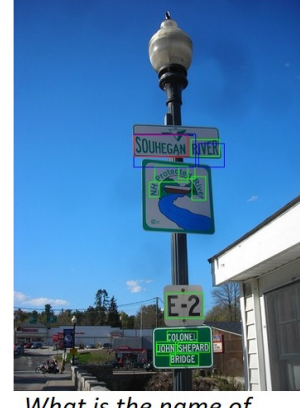

What is the name of the river?

Ours: souhegan river M4C: john shepard

GT: souhegan river IoU: $0.57,0(0.32)$

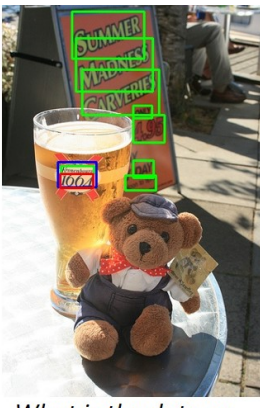

What is the date on the beer glass?

Ours: 1664

M4C: 4.95

GT: 1664 IoU: 0.45

(a)

(c)

(d)

Figure 5: Qualitative examples from ST-VQA dataset. We display predicted answers (Yellow for word generated from OCR and blue for vocabulary) of LaAP-Net and M4C with ground-truth (GT) (GT word not contained in any OCR is printed in gray). Our predicted bounding box is also depicted in the images (blue box) to compare to the GT box (red box). The IoU as an evidence score is also shown in each image. For Figure (c), IoU 0 (0.32) indicates OCR recognition error, 0.32 is the IoU with the GT bounding box.

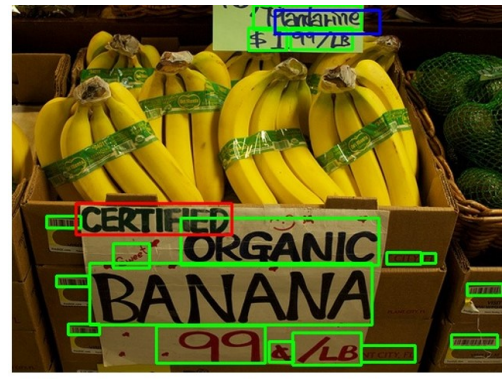

What does the green text word above Organic?

Ours: pandahne

GT: certified

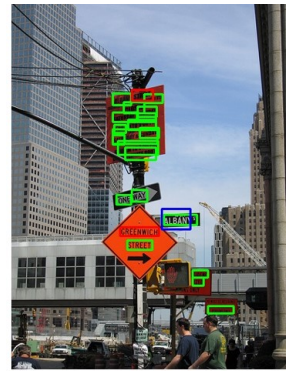

What street is to the right?

Ours: albany

GT: greenwich st.

(b)

Figure 6: Failure examples of our LaAP-Net

\begin{tabular}{|c|c|c|}
\hline Method & Val Acc. & Test Acc. \\
\hline $\begin{array}{c}\text { BLOCK } \\
\text { (Mishra et al., 2019) }\end{array}$ & -- & 42.0 \\
\hline $\begin{array}{c}\text { CNN } \\
\text { (Mishra et al., 2019) }\end{array}$ & -- & 14.3 \\
\hline $\begin{array}{c}\text { Combine+W2V } \\
\text { (Mishra et al., 2019) }\end{array}$ & -- & 48.3 \\
\hline $\begin{array}{c}\text { M4C } \\
\text { (Hu et al., 2020) }\end{array}$ & 63.5 & 63.9 \\
\hline LaAP-Net & $\mathbf{6 3 . 8}$ & $\mathbf{6 4 . 1}$ \\
\hline
\end{tabular}

Table 5: On the OCR-VQA dataset, our LaAPNet model achieves the state-of-the-art result $64.1 \%$ accuracy comparing to $\mathrm{M} 4 \mathrm{C}$ (Hu et al., 2020).

box and answer. In those examples, our proposed localization-aware answer predictor not only generates correct answer, but also predicts exact bounding box(drawn in blue) of the corresponding OCR token. Similar conclusion can be drawn from the result as discussed for TextVQA dataset. In Figure 5(a), our network correctly attends to the middle sign designated by the question, where our reference method M4C fails. In Figure 5(c), our network manages to predict the word 'river' even though it is not recognized by the OCR system. More qualitative examples can be found in the supplementary material.

\subsection{Evaluation on OCR-VQA Dataset}

Unlike TextVQA and ST-VQA that contain "in the wild" images, OCR-VQA dataset consists of 207572 images only of book covers. Thus, the image object modality is less important in OCR-VQA. Moreover, since questions are about the title or author of a book, it is relatively difficult to determine the location. Even so, our model still achieves the state-of-the-art result, $64.1 \%$ accuracy as shown in Table 5.

\subsection{Failure Analysis}

Two failure cases are shown in Figure 6. As discussed in Section 4.2, our model is sensitive to positional instruction in a question. However, in Figure 6(a), the question asks about relative position, which our network does not gain knowledge on. In Figure 6(b), the position "right" is indicated by an arrow, but 
our network locates the road sign on the right of the image. In this case, question answering requires reasoning in addition to text reading function, which we will investigate in our future work.

\section{Conclusion}

This paper proposes a localization-aware answer prediction network (LaAP-Net) for text VQA. Our LaAP-Net not only generates the answer to the question, but also provides a bounding box as an evidence of the answer generated. Moreover, a context-enriched OCR (COR) representation is proposed to integrate object related features. The proposed LaAP-Net outperforms existing approaches on three benchmark datasets for the text VQA task by a noticeable margin with new state-of-the-art performance: TextVQA 41.41\%, ST-VQA 0.485 (ANLS) and OCR-VQA 64.1\%.

\section{References}

Jon Almazán, Albert Gordo, Alicia Fornés, and Ernest Valveny. 2014. Word spotting and recognition with embedded attributes. IEEE transactions on pattern analysis and machine intelligence, 36(12):2552-2566.

Ali Furkan Biten, Ruben Tito, Andres Mafla, Lluis Gomez, Marçal Rusinol, Minesh Mathew, CV Jawahar, Ernest Valveny, and Dimosthenis Karatzas. 2019a. Icdar 2019 competition on scene text visual question answering. In 2019 International Conference on Document Analysis and Recognition, pages 1563-1570. IEEE.

Ali Furkan Biten, Ruben Tito, Andres Mafla, Lluis Gomez, Marçal Rusinol, Ernest Valveny, CV Jawahar, and Dimosthenis Karatzas. 2019b. Scene text visual question answering. In Proceedings of the IEEE International Conference on Computer Vision, pages 4291-4301.

Piotr Bojanowski, Edouard Grave, Armand Joulin, and Tomas Mikolov. 2017. Enriching word vectors with subword information. Transactions of the Association for Computational Linguistics, 5:135-146.

Fedor Borisyuk, Albert Gordo, and Viswanath Sivakumar. 2018. Rosetta: Large scale system for text detection and recognition in images. In Proceedings of the 24th ACM SIGKDD International Conference on Knowledge Discovery \& Data Mining, pages 71-79.

Nicolas Carion, Francisco Massa, Gabriel Synnaeve, Nicolas Usunier, Alexander Kirillov, and Sergey Zagoruyko. 2020. End-to-end object detection with transformers. arXiv preprint arXiv:2005.12872.

Kevin Clark, Urvashi Khandelwal, Omer Levy, and Christopher D Manning. 2019. What does bert look at? an analysis of bert's attention. arXiv preprint arXiv:1906.04341.

Jacob Devlin, Ming-Wei Chang, Kenton Lee, and Kristina Toutanova. 2019. Bert: Pre-training of deep bidirectional transformers for language understanding. In Proceedings of the 2019 Conference of the North American Chapter of the Association for Computational Linguistics: Human Language Technologies, Volume 1, pages 4171-4186.

Peng Gao, Zhengkai Jiang, Haoxuan You, Pan Lu, Steven CH Hoi, Xiaogang Wang, and Hongsheng Li. 2019. Dynamic fusion with intra-and inter-modality attention flow for visual question answering. In Proceedings of the IEEE Conference on Computer Vision and Pattern Recognition, pages 6639-6648.

Chenyu Gao, Qi Zhu, Peng Wang, Hui Li, Yuliang Liu, Anton van den Hengel, and Qi Wu. 2020. Structured multimodal attentions for textvqa. arXiv preprint arXiv:2006.00753.

Yash Goyal, Akrit Mohapatra, Devi Parikh, and Dhruv Batra. 2016. Towards transparent ai systems: Interpreting visual question answering models. arXiv preprint arXiv:1608.08974.

Ronghang Hu, Amanpreet Singh, Trevor Darrell, and Marcus Rohrbach. 2020. Iterative answer prediction with pointer-augmented multimodal transformers for textvqa. In Proceedings of the IEEE Conference on Computer Vision and Pattern Recognition, pages 9992-10002.

Anand Mishra, Shashank Shekhar, Ajeet Kumar Singh, and Anirban Chakraborty. 2019. Ocr-vqa: Visual question answering by reading text in images. In 2019 International Conference on Document Analysis and Recognition, pages 947-952. IEEE.

MSFT-VTI. 2019. textvqa challenge 2019 top entry (post-challenge). https://evalai.cloudcv.org/ web/challenges/challenge-page/244/. 
Shaoqing Ren, Kaiming He, Ross Girshick, and Jian Sun. 2015. Faster r-cnn: Towards real-time object detection with region proposal networks. In Advances in neural information processing systems, pages 91-99.

Ramprasaath R Selvaraju, Michael Cogswell, Abhishek Das, Ramakrishna Vedantam, Devi Parikh, and Dhruv Batra. 2017. Grad-cam: Visual explanations from deep networks via gradient-based localization. In Proceedings of the IEEE International Conference on Computer Vision, pages 618-626.

Amanpreet Singh, Vivek Natarajan, Meet Shah, Yu Jiang, Xinlei Chen, Dhruv Batra, Devi Parikh, and Marcus Rohrbach. 2019. Towards vqa models that can read. In Proceedings of the IEEE Conference on Computer Vision and Pattern Recognition, pages 8317-8326.

Ashish Vaswani, Noam Shazeer, Niki Parmar, Jakob Uszkoreit, Llion Jones, Aidan N Gomez, Łukasz Kaiser, and Illia Polosukhin. 2017. Attention is all you need. In Advances in neural information processing systems, pages 5998-6008.

Xinyu Wang, Yuliang Liu, Chunhua Shen, Chun Chet Ng, Canjie Luo, Lianwen Jin, Chee Seng Chan, Anton van den Hengel, and Liangwei Wang. 2020. On the general value of evidence, and bilingual scene-text visual question answering. In Proceedings of the IEEE Conference on Computer Vision and Pattern Recognition, pages 10126-10135.

Zhou Yu, Jun Yu, Yuhao Cui, Dacheng Tao, and Qi Tian. 2019. Deep modular co-attention networks for visual question answering. In Proceedings of the IEEE Conference on Computer Vision and Pattern Recognition, pages 6281-6290. 


\section{Appendix}

\section{A Implementation and Hyper-parameter Setting}

We use the LoRRA open sourced training environment developed by PyTorch. The question encoder is performed by the first 3 layer of the pre-trained BERT-base model. We also set a maximum decoder step 12 in the answer prediction process. Answer length 12 covers almost all the answers for TextVQA, ST-VQA and OCR-VQA datasets.

We build a fixed vocabulary by using the top 5000 frequent words from the answers of the TextVQA dataset. TextVQA has 10 answers for each question, and the accuracy is measured by the soft-voting for the ten answers. The ST-VQA official metric is average normalized levenshtein similarity (ANLS), which is defined as scores $1-d_{L}\left(a_{\text {pred }}, a_{g t}\right) / \max \left(\left|a_{\text {pred }}\right|,\left|a_{g t}\right|\right)$, (where $a_{\text {pred }}$ and $a_{g t}$ are prediction and ground-truth answers respectively, and $d_{L}$ is the edit distance) averaged over all questions.

The training batch size is set to 64 with 32000 iterations for both TextVQA and ST-VQA dataset. For the OCR-VQA dataset, the batch size is 128 . The best validation accuracy model is used for the test dataset. The entire training takes around 7 hours for TextVQA, 11 hours for ST-VQA and 11 hours for OCR-VQA on 2 Nvida 2080ti GPUs. We summarize the hyper-parameter table for the reproducibility purpose.

\begin{tabular}{|c|c|c|c|}
\hline Input Feature Parameters & Value & Optimizaiton Parameters & Value \\
\hline max question word number K & 20 & optimizer & Adam \\
\hline max detected object number N & 100 & warm-up learning rate factor & 0.2 \\
\hline image object feature dimension & 2048 & max grad L2 -norm for clipping & 0.25 \\
\hline max input OCR token number M & 50 & warm-up iteration & 1000 \\
\hline OCR Fasttext dimension & 300 & max iteration TextVQA & 32000 \\
\hline OCR PHOC dimension & 604 & max iteration ST-VQA, OCR-VQA & 48000 \\
\hline OCR Faster R-RCNN dimension & 2048 & learning rate steps, TextVQA, ST-VQA & 14000,19000 \\
\hline input embedding dimension d & 768 & learning rate steps, OCR-VQA & 28000,38000 \\
\hline input dropout rate & 0.1 & base learning rate & $1 \mathrm{e}-4$ \\
\hline batch size, TextVQA, ST-VQA & 64 & learning rate decay & 0.1 \\
\hline batch size OCR-VQA & 128 & max decoding steps T & 12 \\
\hline Text BERT layer & 3 & LaAP-net Encoder/Decoder Layer & 4 \\
\hline
\end{tabular}

\section{B Additional Qualitative Examples}

In this section, we show additional examples of text VQA answer and predicted bounding box from both TextVQA and ST-VQA datasets to support our claim in the paper. 


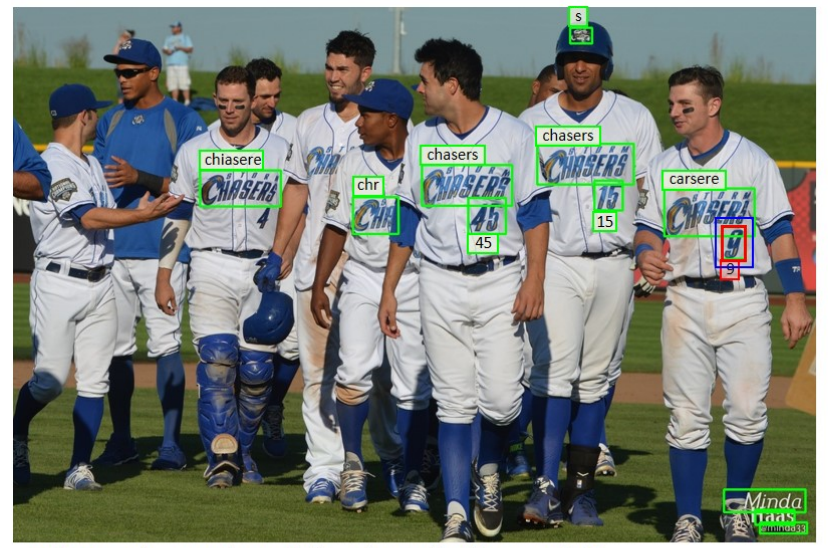

What is the number of the player furthest right?

Ours: 9 GT: 9

(a)

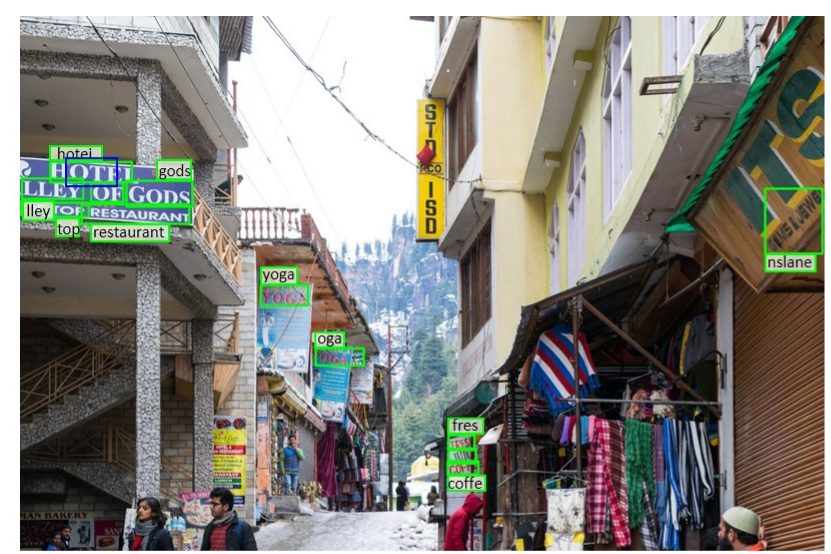

Is there a hotel in this area?

Ours: yes GT: yes

(c)

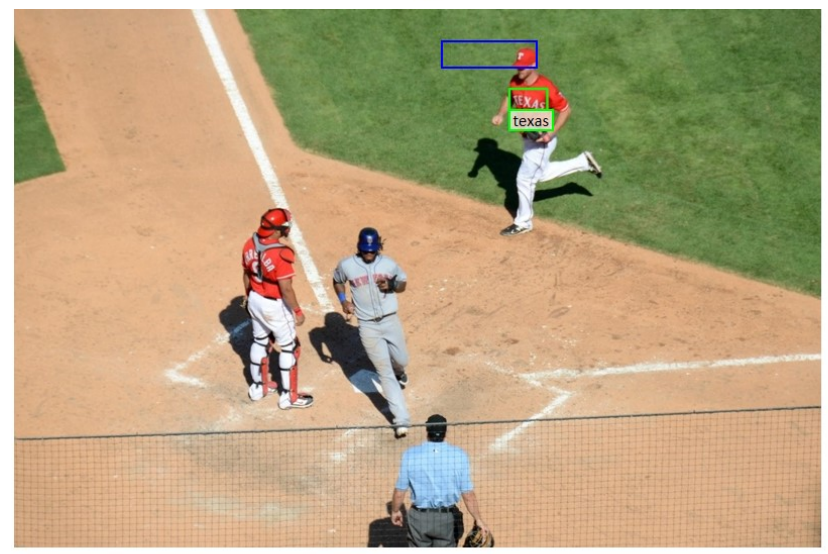

What letter is on the red hat?

Ours: $\mathbf{t}$ GT: $\mathbf{t}$

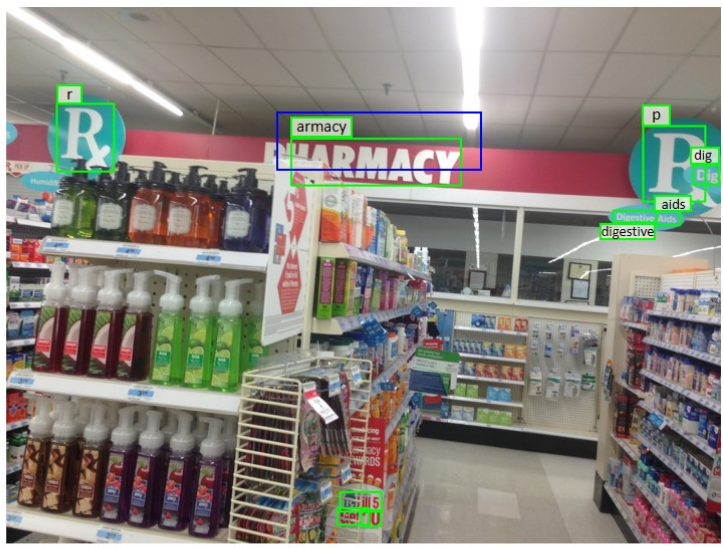

What department is being shown at the store?

Ours: pharmacy GT: pharmacy

(b)

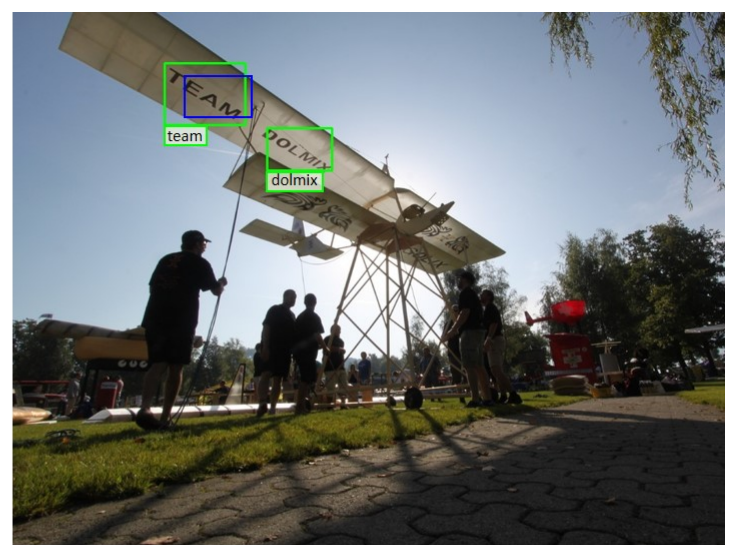

Are they part of team dolmix?

Ours: yes GT: yes

(d)

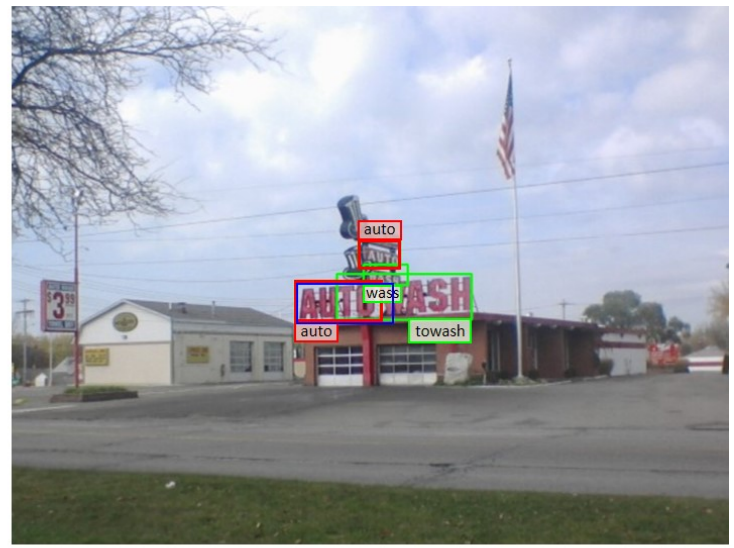

What kind of wash is available?

Ours: auto GT: auto

(e)

(f)

Figure 1: Qualitative examples from TextVQA dataset. We display predicted answers (Yellow for word generated from OCR and blue for vocabulary) of our LaAP-Net and the ground-truth (GT). Our predicted bounding box (blue box) is also depicted in the images to compare to the GT box (red box). Note that some images do not contain GT bounding box while some images contain more than one GT bounding box 


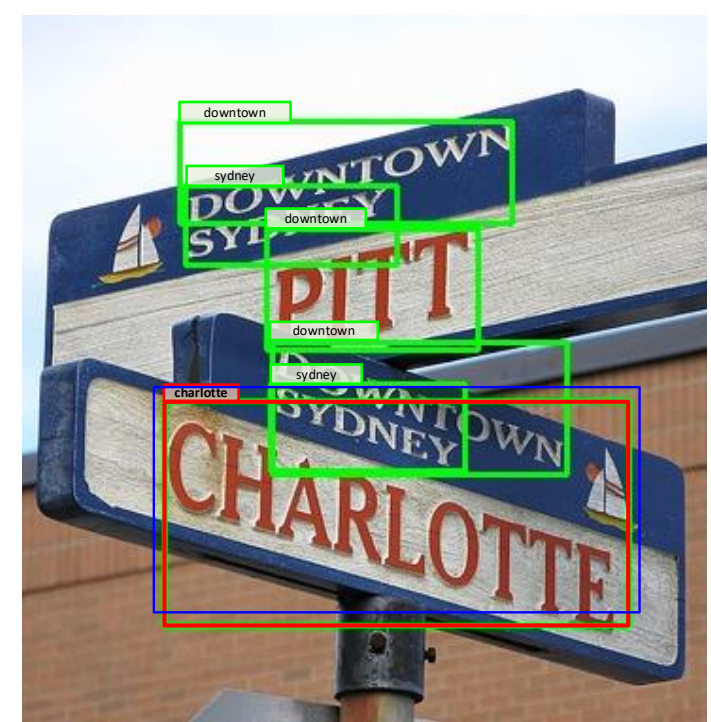

What is the bottom street name?

Ours: charlotte GT: charlotte

(a)

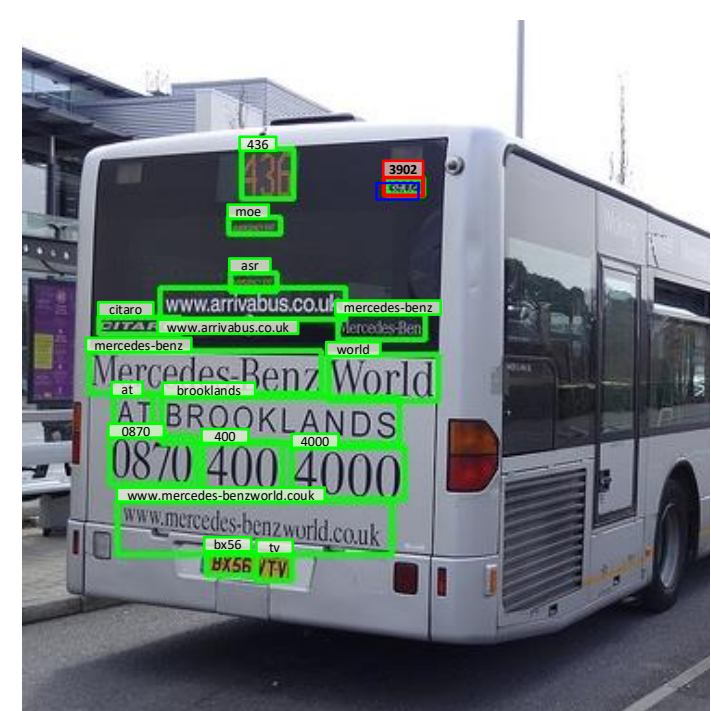

What is the white number on the top right of the back of the bus?

Ours: 3092 GT: 3092

(c)

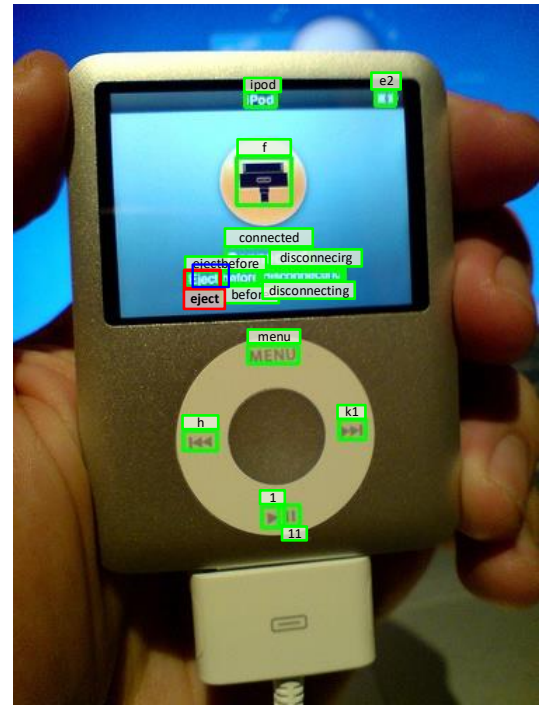

What do you have to do before disconnecting? Ours: eject GT: eject

(b)

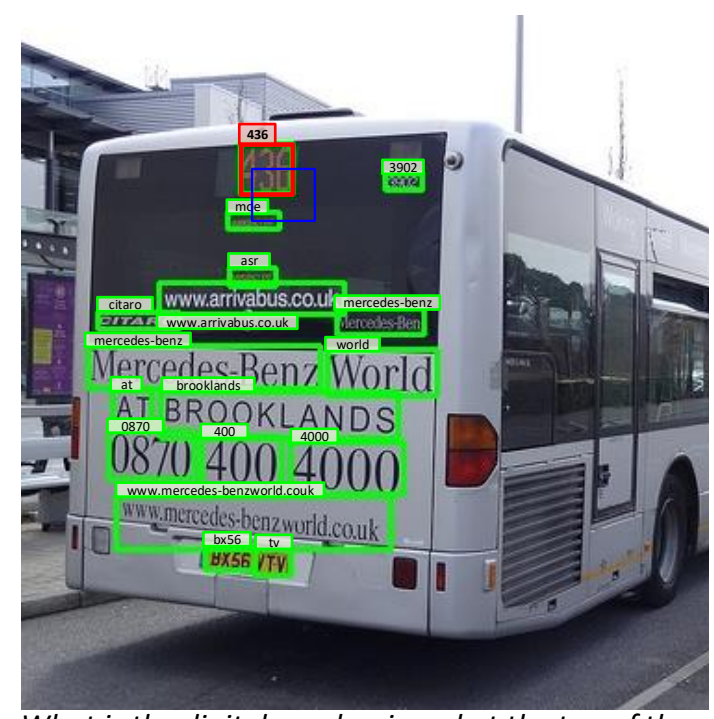

What is the digital number in red at the top of the bus?

Ours: 436 GT: 436

Figure 2: Qualitative examples from ST-VQA dataset. We display predicted answers (Yellow for word generated from OCR and blue for vocabulary) of our LaAP-Net and the ground-truth (GT). Our predicted bounding box (blue box) is also depicted in the images to compare to the GT box (red box). 